\title{
Communication Performance of a Real-Life Wide-Area Low-Power Network Based on Sigfox Technology
}

\author{
Konstantin Mikhaylov*†, Martin Stusek*, Pavel Masek*, Radek Fujdiak* ${ }^{* \ddagger}$ Radek Mozny*, \\ Sergey Andreev ${ }^{\S}$, and Jiri Hosek* \\ *Department of Telecommunications, Brno University of Technology, Brno, Czech Republic \\ ${ }^{\dagger}$ Center for Wireless Communications, University of Oulu, Oulu, Finland \\ ${ }^{\ddagger}$ Technical University of Ostrava, Ostrava, Czech Republic \\ $\S$ Unit of Electrical Engineering, Tampere University, Tampere, Finland
}

\begin{abstract}
In this paper, we study real-world performance of Sigfox, which is one of the most mature Low-Power WideArea Network (LPWAN) technologies that operate in unlicensed frequency bands. During an extensive measurement campaign conducted over three months in the city of Brno, Czech Republic, we assessed the communication performance and the radio channel properties in 311 different test locations. We observed that despite the challenging natural landscape and urban environment of the test area, more than $94 \%$ of the packets sent were received successfully, with at least one packet delivered from 297 out of 311 tested locations. Our results also reported experiment-based radio channel and signal-to-noise characterization as well as provided insights into the efficiency of two crucial mechanisms used by Sigfox to improve the packet delivery - packet repetition and multi-gateway reception. Finally, we employed our experimental data to understand the efficiency of two non-fingerprint localization methods based on received signal strength indicator in a practical Sigfox network.

Index Terms-LPWAN, Sigfox, experiment, performance, measurement, localization, triangulation, IoT
\end{abstract}

\section{INTRODUCTION}

The XXth century has brought remarkable progress in human-centered wireless connectivity. The first decades of the XXIst century have introduced new connectivity actors to the stage - the machines. They are becoming excessively diverse with respect to their appearances, capabilities, resources, applications, targets, motivations, and communication needs. To address these needs and to enable the formation of a harmonized connectivity landscape for all machines the Internet of Things (IoT) - novel wireless communication technologies [1] have been developed in the past years.

Specifically, to effectively address the demands of noncritical IoT applications with infrequent data transmissions and across wide deployment areas, e.g., smart meters or tracking devices, to name a few, the Low Power Wide Area Network (LPWAN) [1], [2] technologies have been introduced. Based on the employed frequency spectrum, the LPWAN technologies of today can be divided into two major groups. The Narrow-Band IoT (NB-IoT) and Long-Term EvolutionMachine (LTE-M) solutions are developed by the 3rd Generation Partnership Project (3GPP) and originate from the well- known mobile broadband technology named LTE. They benefit from operating in the licensed frequency bands. In contrast to these, a much broader and more diverse group of LPWAN options operate in the unlicensed bands.

The two unlicensed-band LPWAN technologies dominating the today's market are Sigfox and LoRaWAN [3]. Both of these solutions (i) follow the star-of-stars network topology (composing the end devices (EDs), gateways (GWs), and server/cloud), (ii) use Aloha-based media access control (MAC) with random selection of a frequency channel (or carrier frequency in the case of Sigfox), (iii) do not imply any association between a GW and EDs, and (iv) operate in sub$\mathrm{GHz}$ Industrial, Scientific, and Medical (ISM) band $(868 \mathrm{MHz}$ in Europe and $915 \mathrm{MHz}$ in the US). Among the key differences between the two technologies are (i) different modulations and bandwidths, (ii) a number of available modulation and coding schemes (MCS) and MCS adaptation mechanisms, and (iii) limitations and business models.

Specifically, LoRa modulation enabling the LoRaWAN solution spreads the signal over the band of $125 \mathrm{kHz}$, while Sigfox signals are ultra-narrowband and occupy the frequency band of 100 to $600 \mathrm{~Hz}$. Moreover, LoRaWAN network allows adapting the transmit power and MCS (i.e., the spreading factor parameter) based on the radio channel conditions, while the transmit power and modulation for Sigfox are fixed. Both of these approaches have their pros and cons. For the EDs located closer to the GWs, LoRaWAN helps reduce their energy consumption and increase the throughput via dedicated signaling mechanisms. Meanwhile, Sigfox devices do not require any channel information, which is more predictable concerning the energy consumption and throughput. Finally, regarding the business model, LoRaWAN supports both operator-deployed public networks and user/applicationspecific private networks. All of the Sigfox deployments are consolidated into a global public network, with a single operator covering each geographical zone.

Even though Sigfox has been in commercial roll-outs for longer (since 2012 - 2013) than LoRaWAN (since 2015), which is owing to the possibility of a private network deployment by 
any party, the LoRaWAN technology has been studied much more extensively. Specifically, aside from numerous surveys and overviews comparing the technical solutions and their performance, while speculating on the use cases of different LPWAN options, only a limited number of works addressing Sigfox are available. The energy consumption model of a Sigfox device has been reported in [4]. The scalability of a Sigfox network has been investigated using analytical methods by the authors of [5]-[7]. The localization capabilities of Sigfox based on the received signal strength indicator (RSSI) fingerprinting have been studied in [8]-[10]. The experience of developing applications over Sigfox has been summarized by the authors of [11] and [12].

However, the volume of data about the real-world performance of large-scale Sigfox networks and the effects pertaining to such deployments are insufficiently covered in the current literature. To address this gap, we here report on the key findings of our extensive measurement campaign conducted in the city of Brno, Czech Republic, over the period of three months. During this time, the communication performance of over three hundred test locations was characterized. The results of the subsequent analysis reveal the practical performance of a large-scale Sigfox network, which constitutes the major contribution and the main novelty of this paper. The secondary contributions are our results demonstrating the performance of localization based on the RSSI data from multiple Sigfox gateways and a propagation model determined from our experimental data.

The rest of this paper is organized as follows. In Section II, we review the relevant aspects of the Sigfox technology. In Section III, we outline our experimental configurations and procedures. In Section IV, we first report and discuss our key measurement-specific findings, and then present the results of RSSI-based localization. Finally, Section V summarizes our messages and concludes the paper.

\section{Sigfox Technology BACKground}

A Sigfox network is composed of the EDs, the GWs, and the cloud core. The EDs transfer their data wirelessly to the GWs, which forward the said data via an Internet connection into the cloud, thus making them accessible to a user through the Application Program Interface (API). The underlying security solution is based on symmetric keys, which are given to the EDs during their manufacturing or deployment, and registered in the cloud core prior to the ED operation. Payload encryption is an optional feature provided at an extra cost.

Fig. 1 illustrates a typical Sigfox communication procedure and the respective frame structures. The communication between an ED and a GW is always initiated by the ED. The latter can start its transmission at any instant of time given that it does not violate the limitations of the radio channel utilization imposed by the local regulatory authorities and the Sigfox operators. The maximum user data payload of a single uplink packet is limited to 12 bytes [13]. The uplink transmissions are subject to Differential Binary Phase-Shift Keying (D-BPSK) and are sent at the rate of 100 bps (Radio
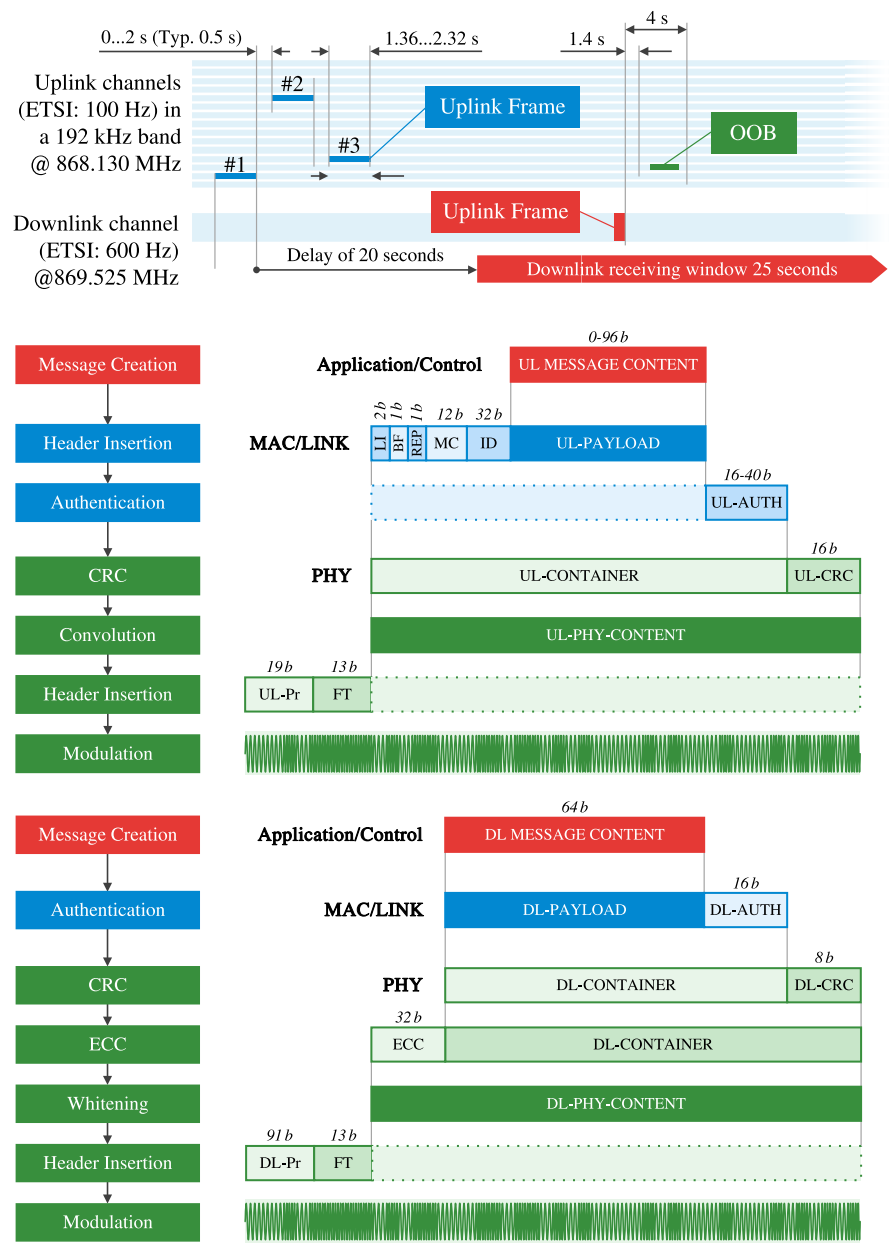

Fig. 1. Sigfox bi-directional communication procedure in multi-frame mode and respective frame structures.

Configurations (RCs) 1, 3, 5, and 6 - i.e., EU, Middle East, Africa, and Asia [14]) or 600 bps (RCs 2 and 4 - both Americas, Australia, and some Asia-Pacific regions [14]). The carrier frequency is selected randomly within the operating band (typically, $200 \mathrm{kHz}$ ) by maintaining a gap from the carrier used for the previous uplink transmission [14]. The duration of a frame (depending on the size of the payload) is 1.12 to 2.08 seconds for RCs $1,3,5$, and 6 , and between 187 and $347 \mathrm{~ms}$ for RCs 2 and 4 [14].

An ED can send a packet either once (so-called singleframe transmission mode) or repeat it several times (typically three times, named multi-frame transmission mode), each time selecting its carrier frequency randomly [15] and using different convolutional codes [14]. The delay between the packet repetitions may range from 4 uplink symbol durations (i.e., $40 \mathrm{~ms}$ for $\mathrm{EU}$ and $6.7 \mathrm{~ms}$ for the US) to two seconds (RC 1, 2, 4, and 5) [16]. Based on their maximum transmit power in the uplink, Sigfox EDs are subdivided into four classes labeled $0 \mathrm{U}(12-16 \mathrm{dBm}$ for $\mathrm{EU}$ or $20-24 \mathrm{dBm}$ for the US) to $3 \mathrm{U}$ (below $0 \mathrm{dBm}$ for $\mathrm{EU}$ or $5 \mathrm{dBm}$ for the US) [15], [16]. It is expected that for most of the devices, the transmit power remains constant throughout their lifetime [15]. 
Bi-directional communication is an optional feature in Sigfox (named "B-procedure", in contrast to "U-procedure" for uplink-only; the procedure is selected on a per-message basis). The ED initiates a B-procedure by setting a special flag in the uplink frame header [14]. Twenty (or 19, for RC 3 and 5) seconds after the end of the first uplink packet transmission, the $\mathrm{ED}$ opens a receive window with 25 (or 33.5 , for $\mathrm{RC} 3$ and 5) seconds of duration. The frequency at which the ED listens on is calculated based on the carrier of the first uplink frame and the region-specific offset [14]. Note that in the case of using a multi-frame transmission under "B-procedure", the carrier frequency for the second and third retransmissions is selected by introducing a dedicated region-specific shift from the carrier frequency of the first retransmission.

For all RCs, downlink transmissions are subject to Gaussian frequency-shift keying (GFSK) with the rate of $600 \mathrm{bps}$. The maximum size of user data is limited to 8 bytes. Upon a successful reception of a downlink message, the ED is required to send an uplink confirmation control message [14] carrying the basic information about the device status and the RSSI for the downlink message. The transmission needs to start within 1.4 to 4 seconds from the downlink packet reception time by using a single-frame mode and a random carrier frequency.

\section{Measurement Setup and Procedure}

Our measurements have been carried out in the city of Brno, Czech Republic, from February to April 2019. The size of the test area was approximately $12 \mathrm{~km}$ north to south and $24 \mathrm{~km}$ west to east. The tests were conducted in 311 different locations ${ }^{1}$, which were primarily public transportation stop points. The test area was served by multiple Sigfox SBS-T3 GWs, having the sensitivity of up to $-142 \mathrm{dBm}$ and operating in $868 \mathrm{MHz}$ band. As ED equipment, we used Adeunis RF Sigfox field test device. The transmit power was configured to $14 \mathrm{~dB}$ $(25 \mathrm{~mW})$, and we employed $0 \mathrm{dBi}$ integrated omnidirectional wire-antenna. The device was sending its data in the multiframe mode via U-procedure.

During our measurements, the test device was positioned at each test point approximately one meter above the ground level, away from buildings and other obstacles. The device was powered up and made to transmit several radio frames. The timestamps of the beginning and the end of each experiment as well as the accurate location of every test point according to the global navigation satellite system (GNSS) receiver were recorded. After the tests, per-packet data (including the radio parameters measured by the GWs, timestamps, and information about the number of repetitions received) were downloaded from the Sigfox cloud and processed. Note that for each packet, the information from at most four GWs has been made available. The locations of the GWs were determined from the map with the accuracy of about $\pm 50 \mathrm{~m}$.

\footnotetext{
${ }^{1}$ The map of measurement points is available via: https://drive.go ogle. com/open?id=1_m9OAu 4 IKJqdz jAyo0AD1bnRBP rbbJrZ\&u $\mathrm{sp}=$ sharing
}

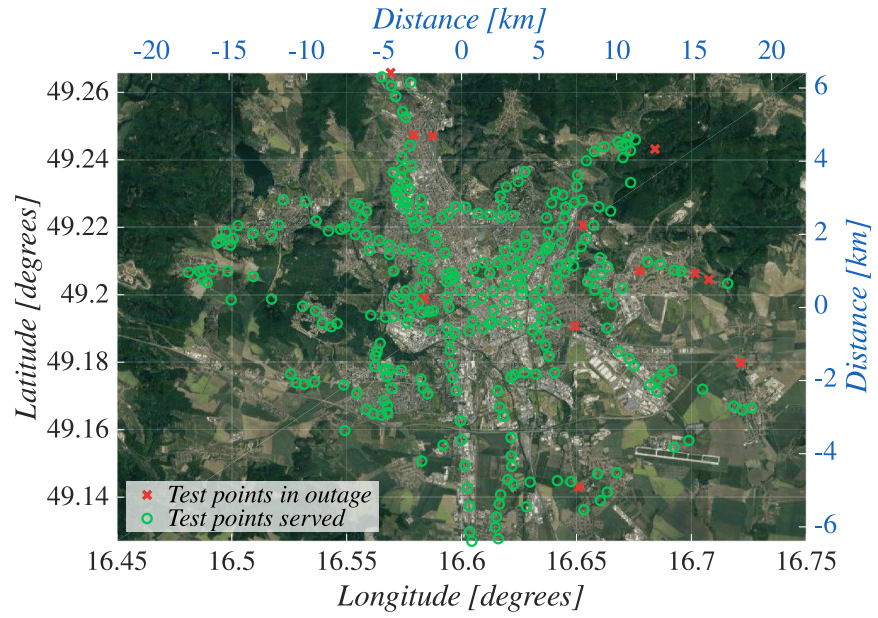

Fig. 2. Map of experiment area demonstrating test locations and whether any packets have been received from a location.

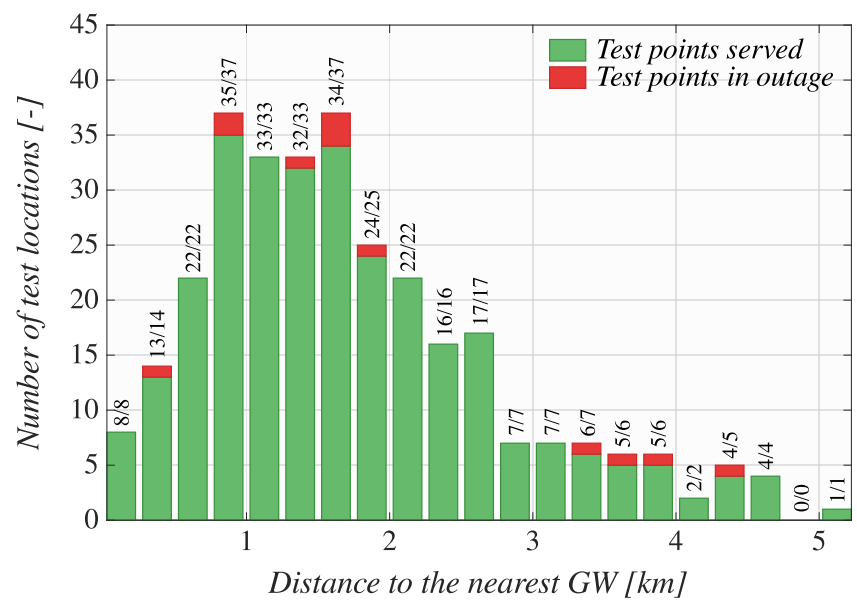

Fig. 3. Effect of proximity to the nearest GW on outage probability.

\section{SElected Results}

\section{A. Main Experimental Findings}

Fig. 2 illustrates the test area by depicting the location of each test point. In green, we mark the locations from which at least one packet has been received, and in red we highlight the ones from which no data has been delivered. In what follows, the latter ones are denoted as being in outage. Overall, the data from 297 out of 311 test locations $(95.5 \%)$ was received and the cumulative packet delivery ratio (PDR) was $94.79 \%$.

Fig. 3 displays the outage probability vs. the distance to the nearest GW. None of the test points were located at the distance of over $5.25 \mathrm{~km}$ and many were within $2-3 \mathrm{~km}$ from the proximate $\mathrm{GW}$. One of the test points in outage was located within $500 \mathrm{~m}$ from its closest $\mathrm{GW}$, and most points in outage were $750-2000 \mathrm{~m}$ away from their GWs. Among the possible reasons for this behavior are non-line-of-sight propagation conditions and interference.

Figs. 4 and 5 illustrate the maximum RSSI of the signals received from different locations and demonstrate the impact of distance on the RSSI for each received packet, respectively. For most of the locations, the RSSI level remained between 110 and $-130 \mathrm{dBm}$, and only from one test point the signal was 


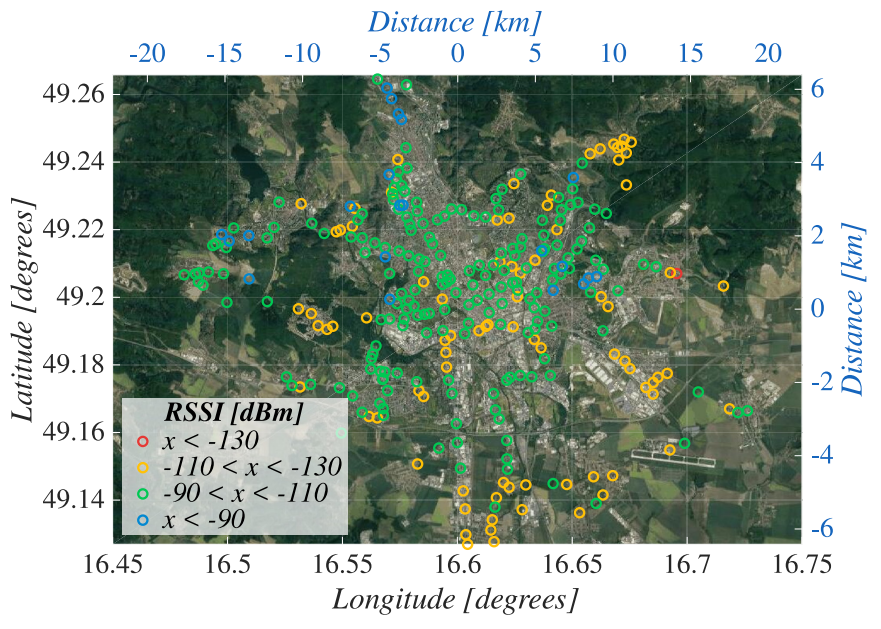

Fig. 4. Highest RSSI for signals from different test locations.

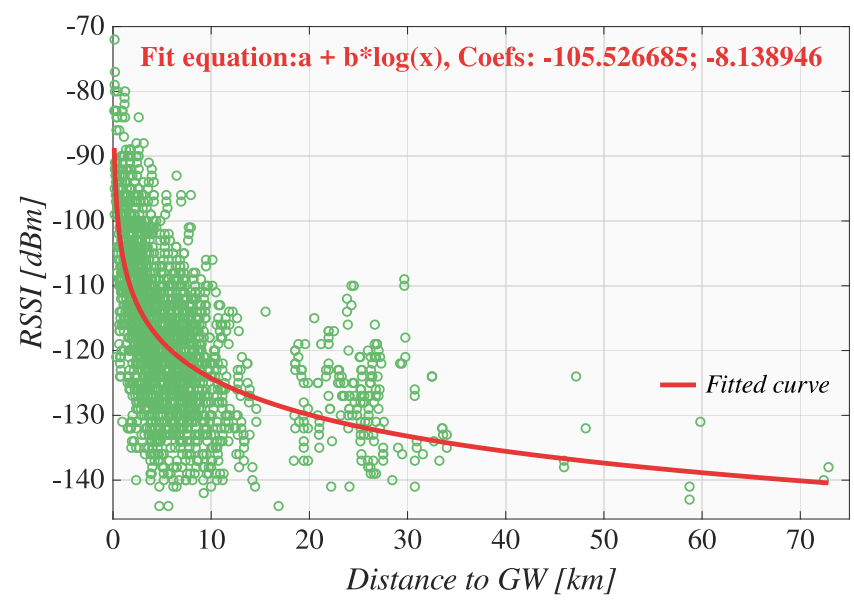

Fig. 5. RSSI vs. distance for each received radio packet.

below $-130 \mathrm{dBm}$. The maximum and the minimum values of the RSSI observed in our experiments were -72 and $-144 \mathrm{dBm}$, respectively. One can also notice a considerable variation in the RSSI values for transmissions within the range of 0.5 to $10 \mathrm{~km}$. These are likely caused by the fact that at these distances, both line-of-sight and non-line-of-sight transmissions are received by the GWs. From Fig. 5, one can learn that several packets were received by the GWs located at the distance of over $70 \mathrm{~km}$ away. Note that Fig. 5 also depicts the coefficients for a least-squares fit using a logarithmic curve for the radio channel model based on our experimental data.

The effect of distance to the GW receiving a packet on the signal-to-noise ratio (SNR) measured by this GW and another effect of the relation between the SNR and the RSSI are highlighted in Figs. 6 and 7, respectively. For all the received packets, the SNR was above $6 \mathrm{~dB}$. The maximum SNR level was around $16 \mathrm{~dB}$. Considering the impact of RSSI on SNR, one can expect that for the very high RSSI values, the SNR is also typically large. However, for the radio packets with the RSSI of below $-90 \mathrm{dBm}$, the SNR may vary significantly.

Spatial diversity is one of the key mechanisms for increasing the reliability of packet delivery in Sigfox. Fig. 8 shows how many GWs on average have received a transmission from each

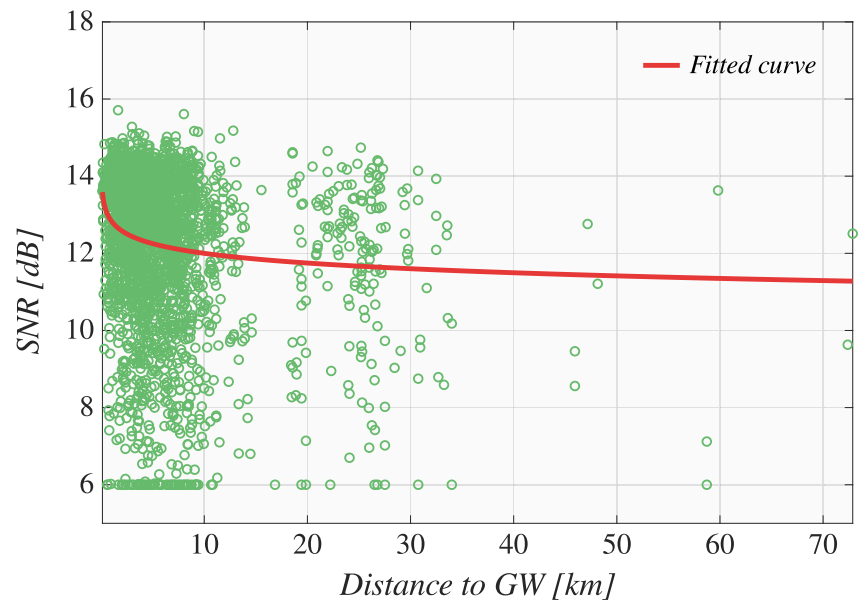

Fig. 6. SNR vs. distance for each received radio packet.

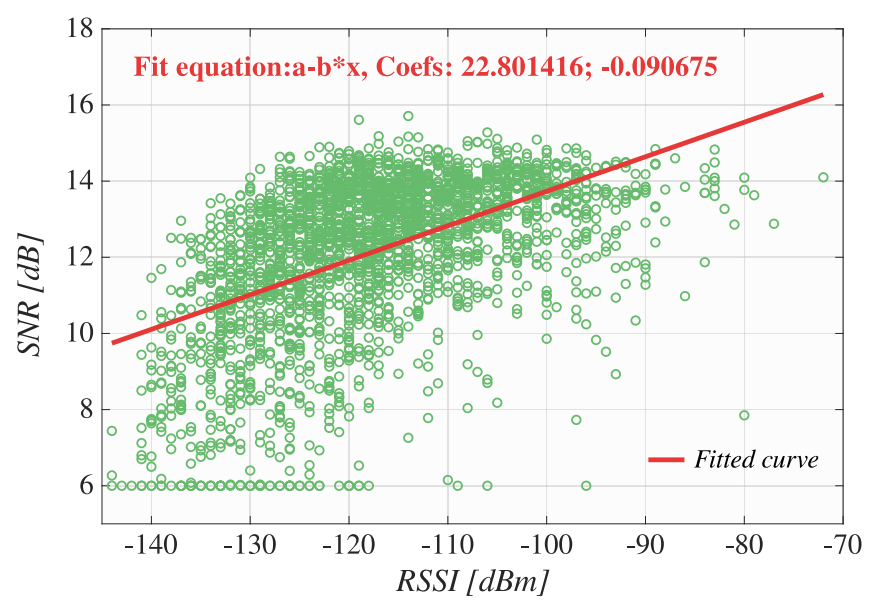

Fig. 7. SNR vs. RSSI for each received radio packet.

test location. Fig. 9 details these results further by highlighting the share of packets received by various numbers of GWs. One can see that $71.4 \%$ of the packets were received by at least four GWs. The mean number of GWs receiving a packet throughout the entire experimental campaign was 3.56.

Finally, Figs. 10 and 11 display the maximum number of packet repetitions received from each test location and with respect to the distance to the nearest $\mathrm{GW}$, correspondingly. One can infer that at almost any location there were packets with all three repetitions received. However, there was also a substantial number of packets for which only one repetition has been used, even for the locations near the GWs.

\section{B. On RSSI-based Localization}

Given that many IoT applications either require or may benefit from having location-specific information, we use our experimental data to assess how accurately one may determine a test point's location based on the RSSI data. Given the limited amount of experimental data, in what follows, we exclusively consider non-fingerprint based methods. Note that Sigfox provides native support for geolocation based on RSSI and machine-learning [18]. Since the details of these features 


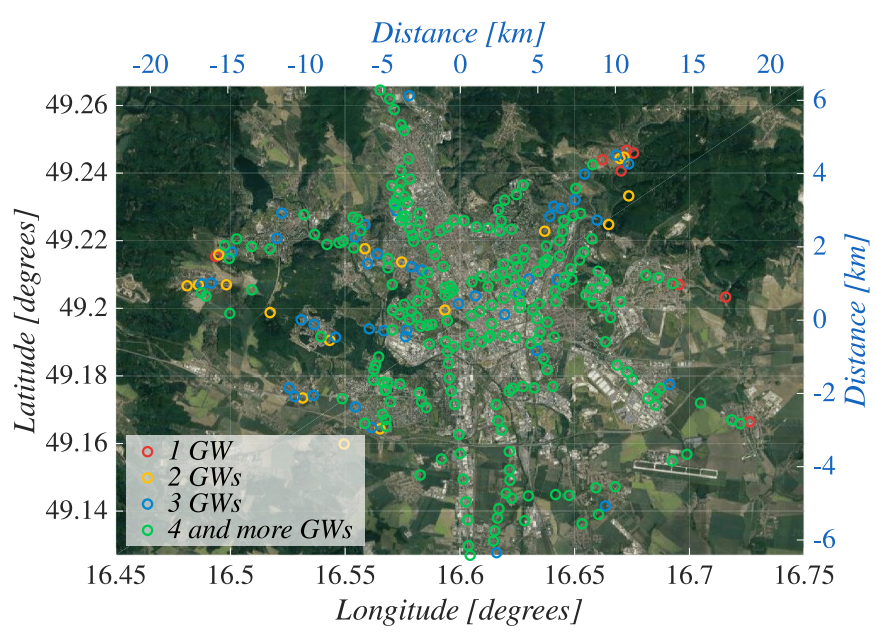

Fig. 8. Number of GWs receiving packets from different test locations.

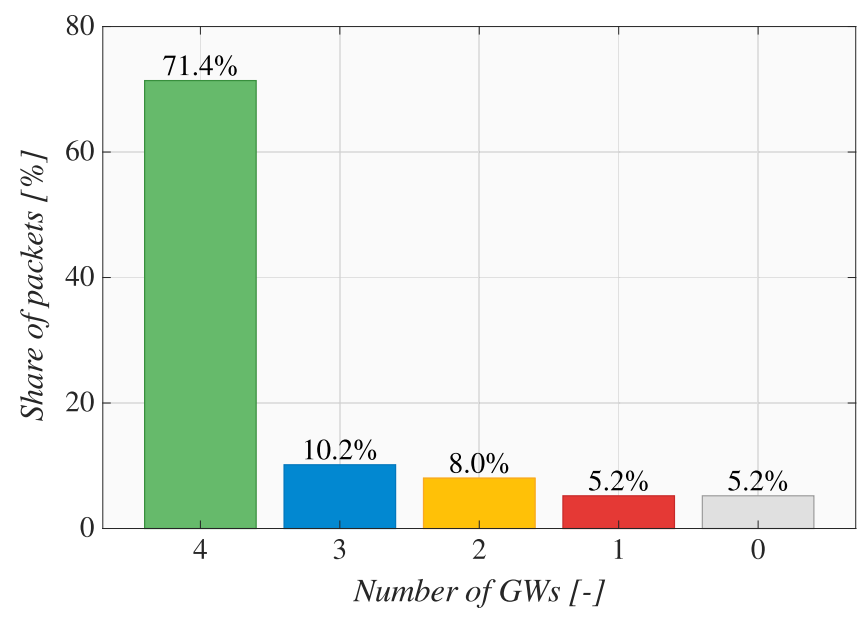

Fig. 9. Share of packets received by various numbers of GWs.

are not available, we here explore the performance of two conventional methods.

The first method under test assigns the coordinates of the GW having the highest RSSI as an estimate of the test point location. Despite being straightforward, this approach works in the case where at least one $\mathrm{GW}$ has received a packet. The mean localization error demonstrated by the method at hand over all the packets was $3.54 \mathrm{~km}$, while the minimum and the maximum errors were 0.13 and $25.5 \mathrm{~km}$, respectively. For $80 \%$ of the packets, the error was below $5.28 \mathrm{~km}$.

The second examined method is the conventional triangulation. First, the packets that have been received by at least three GWs were identified. If four GWs received a packet, for the purposes of triangulation, we used three GWs with the highest RSSI. Further, we utilized our experimental channel model (see the equation in Fig. 5) to determine the distance to each GW and aimed at estimating the test point location based on these data. In total, 610 out of 709 packets have been received by at least three GWs, and only for 38 packets the triangulation converged.

The resulting mean error was $6.31 \mathrm{~km}$, while the minimum and the maximum errors were 1.40 and $14.49 \mathrm{~km}$, respectively.

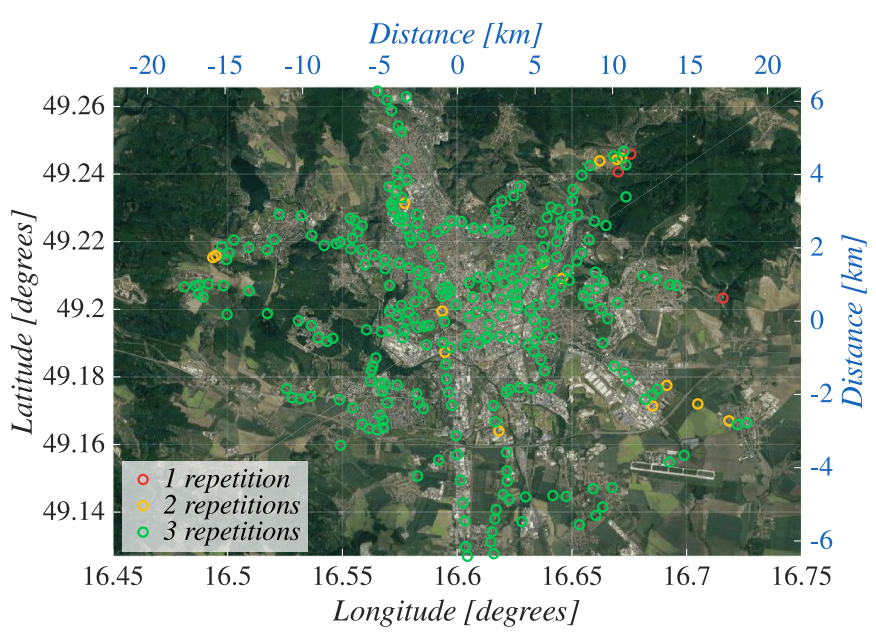

Fig. 10. Maximum number of packet repetitions received from test points.

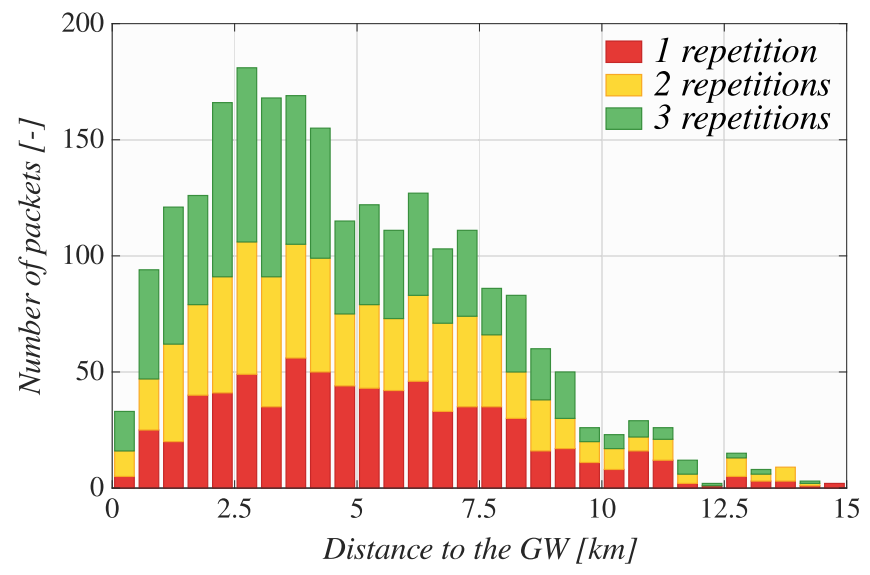

Fig. 11. Effect of distance between a test point and its GW on number of repetitions received.

For $80 \%$ of the packets, the maximum error was below $8.52 \mathrm{~km}$. To make a comparison of the two methods fair, we then applied the first approach only to those packets, for which triangulation produced meaningful results. The mean, minimum, maximum, and $80-\%$ percentile error for this subset of packets constituted $5.2 \mathrm{~km}, 0.63 \mathrm{~km}, 18.88 \mathrm{~km}$, and $7.16 \mathrm{~km}$, respectively. These results are comparable with the performance of the initial version of Sigfox localization algorithms (10 km for $80 \%$ of the packets [18]).

\section{Discussion AND CONCLUSIONS}

The key contributions and novelty of this paper are in the presented results of an extensive measurement campaign that reports on the performance of a real-world multi-GW Sigfox network. The experiments were carried out over three months in 311 different locations around the city of Brno, Czech Republic. They allow one to better understand the behavior, capabilities, and limitations of a practical Sigfox deployment and thus might become of interest to a broad range of stakeholders, including the network planners, application developers, and systems engineers. For academia, these results 
represent valuable ground-truth information, which is scarce in today's literature.

Below, we summarize our main results and observations as well as provide further clarifications:

- The communication reliability observed during our experiments was rather high $-94.79 \%$ of the packets sent were received, and we have acquired data from $95.5 \%$ test locations. On average, a packet was received by 3.56 GWs. Based on these results, we can conclude that Sigfox is a promising solution for a wide spectrum of low data packet loss and delay tolerant applications. However, it may not suit all the use cases that demand high reliability constraints.

- The measurements were conducted in an urban area, which is covered by several Sigfox GWs. For each test location, at least one Sigfox GW was present within the range of $5.25 \mathrm{~km}$, and for the majority of the test points, it appeared within $2.5 \mathrm{~km}$ range. However, during our experiments, we also observed the reception of a few packets from the distances exceeding $70 \mathrm{~km}$.

- The experimental radio channel and SNR models were contributed in this work. Also, we observed that the variation of RSSI/SNR for the test locations at the same distance from a GW is often very significant. When the distance remains between 1 and $30 \mathrm{~km}$, this difference is especially large. A plausible reason here is the fact that at these distances the packets traveling over the direct lineof-sight path and suffering from refraction are received by the GWs.

- Using the coordinates of the GWs with the best RSSI as an estimate of device location resulted in the mean error of $3.54 \mathrm{~km}$, while having the error of below $5.28 \mathrm{~km}$ for $80 \%$ of the cases. The triangulation method using the data from three GWs with the best RSSI and our experimental channel model converged for only $5.3 \%$ of the packets. The mean error in this approach was $6.31 \mathrm{~km}$, while for $80 \%$ of the packets, the maximum error was below $8.52 \mathrm{~km}$. Note that most of our measurements were carried out in an urban environment served by multiple GWs. In suburban or rural environments, the results may be different.

We expect that the features of the natural landscape and the urban layout of the test area (i.e., hills and mid-rise buildings) have impacted the propagation of the radio signal, thus affecting our obtained results. Also, the same radio frequencies might have been used by other radio communication and LPWAN technologies, including, e.g., private and commercial LoRaWANs. It is likely that they have created interference during the measurements. Note that all our measurements have been conducted outdoors and on the ground level; communication in more challenging environments (e.g., building basements) was not considered. However, the RSSI levels observed in many test locations were rather high, thus offering a reasonable margin for combating additional attenuation introduced by, e.g., building walls.
Among our further steps, we plan to arrange experimental measurements of long-term fluctuations in practical Sigfox radio channels, as well as produce a more focused characterization of how the deployment environment (e.g., indoor or sub-terrain layouts) affects the communication performance. Another important challenge is the optimization of Sigfox device operation, including, e.g., switching between singleand multi-frame modes. Analysis of data traffic to enable GWs to save energy by entering a sleep mode might also be of interest.

\section{ACKNOWLEDGMENT}

For this research, the infrastructure of the SIX Center was used. This paper is based upon the support of international mobility project MeMoV, No. CZ.02.2.69/0.0/0.0/16_027/00083710 funded by European Union, Ministry of Education, Youth and Sports, Czech Republic, and Brno, University of Technology. The work of K. Mikhaylov has been supported by the Academy of Finland 6G Flagship (grant 318927).

\section{REFERENCES}

[1] G. A. Akpakwu, B. J. Silva, G. P. Hancke, and A. M. Abu-Mahfouz, "A Survey on 5G Networks for the Internet of Things: Communication Technologies and Challenges," IEEE Access, vol. 6, p. 3619, 2018.

[2] U. Raza, P. Kulkarni, and M. Sooriyabandara, "Low Power Wide Area Networks: An Overview," IEEE Commun. Surv. \& Tut., vol. 19, no. 2, pp. 855-873, Secondquarter 2017.

[3] J. Blackman, "LPWA matchup - LoRaWAN vs Sigfox vs NB-IoT vs LTE-M: trading blows (round 3)", available: https://enterpriseiotinsights. com/20190822/channels/fundamentals/lpwa-matchup-round-three

[4] C. Gomez, J. Veras, R. Vidal, L. Casals, and J. Paradells, "A Sigfox Energy Consumption Model," Sensors, vol. 19, no. 3, p. 681, Feb. 2019

[5] A. Lavric, A. I. Petrariu and V. Popa, "Long Range SigFox Communication Protocol Scalability Analysis Under Large-Scale, High-Density Conditions," IEEE Access, vol. 7, pp. 35816-35825, 2019.

[6] Y. Mo, C. Goursaud, and J. Gorce, "Multiple Base Stations Diversity for UNB Systems: Theoretical Analysis and Performances," in Proc. Int Symp. Netw., Comp. and Commun., Rome, 2018, pp. 1-6.

[7] Y. Mo, C. Goursaud, and J. Gorce, "On the Benefits of Successive Interference Cancellation for Ultra Narrow Band Networks: Theory and Application to IoT," in Proc. Int. Conf. Commun., Paris, 2017, pp. 1-6.

[8] T. Janssen, M. Aernouts, R. Berkvens, and M. Weyn, "Outdoor Fingerprinting Localization Using Sigfox," in Proc. Int. Conf. Indoor Positioning and Indoor Navigation, Nantes, 2018, pp. 1-6.

[9] M. Aernouts, B. Bellekens, R. Berkvens and M. Weyn, "A Comparison of Signal Strength Localization Methods with Sigfox," in Proc. Int. Workshop Positioning, Navigation and Commun., Bremen, 2018, p. 1.

[10] G. G. L. Ribeiro et al., "An Outdoor Localization System Based on SigFox," in Proc. IEEE Vehicular Technol. Conf., Porto, 2018, pp. 1-5.

[11] Y. Chung, J. Y. Ahn and J. Du Huh, "Experiments of A LPWAN Tracking(TR) Platform Based on Sigfox Test Network," in Proc. Int. Conf. Inf. Commun. Techn. Convergence, Jeju, 2018, pp. 1373-1376.

[12] L. Joris et al. "An Autonomous Sigfox Wireless Sensor Node for Environmental Monitoring," IEEE Sens. Let., vol. 3, no. 7, pp. 1-4, July 2019 .

[13] Sigfox: Radio Technology Keypoints, available: https://www.sigfox. com/en/sigfox-iot-radio-technology

[14] Sigfox, Sigfox Connected Objects: Radio Specifications, Ref.: EPSPECS Rev.: 1.3, Feb. 2019.

[15] Sigfox, Sigfox Device Cookbook, Nov. 2018.

[16] J. Koepp, N. Mahmud, D. Bues, and A. Mandel, Rohde-Schwarz: Characterization of Sigfox Devices from Lab to Production Line, Application Note, 2.2018-1MA294_2e.

[17] Sigfox: Downlink Information, available: https://support.sigfox.com/ docs/downlink-information

[18] Sigfox Geolocation Flyer, available https://www.sigfox.com/sites/ default/files/Sigfox\%20Geolocation\%20Flyer\%20Digital\%20A4.pdf 